\title{
FICHTE ET LA PUISSANTE IMPUISSANCE DU LANGAGE
}

\author{
Luis Fellipe Garcia
}

Centre Sèvres | «Archives de Philosophie»

2020/1 Tome 83 | pages XIX à XXXII

ISSN 0003-9632

Article disponible en ligne à l'adresse :

https://www.cairn.info/revue-archives-de-philosophie-2020-1-page-XIX.htm

Distribution électronique Cairn.info pour Centre Sèvres.

(c) Centre Sèvres. Tous droits réservés pour tous pays.

La reproduction ou représentation de cet article, notamment par photocopie, n'est autorisée que dans les limites des conditions générales d'utilisation du site ou, le cas échéant, des conditions générales de la licence souscrite par votre établissement. Toute autre reproduction ou représentation, en tout ou partie, sous quelque forme et de quelque manière que ce soit, est interdite sauf accord préalable et écrit de l'éditeur, en dehors des cas prévus par la législation en vigueur en France. Il est précisé que son stockage dans une base de données est également interdit. 


\section{Fichte et la puissante impuissance du langage}

\section{Luis Fellipe Garcia}

Fondation Alexander von Humboldt, Ludwig-Maximilians-Universität München

« D 'après mon plan, je ne ferai [...] rien que chercher à rendre populaires et, à travers mon éloquence, rendre efficaces [wirksam] sur le cœur des hommes les principes [de la philosophie kantienne] ${ }^{1}$ ", annonçait Fichte dans une lettre à sa future épouse à la fin de l'année 1790, soit environ trois ans avant d'avoir eu l'intuition de son propre projet philosophique, inspiré notamment par ses lectures des nouveaux sceptiques qui, comme l'Enésidème, auraient « renversé de fond en comble les principes de la philosophie kantienne ${ }^{2}$ ». Si, à la suite de la formulation de sa Wissenschaftslehre, son rapport à l'œuvre du maître de Königsberg s'est transformé au point où l'on pourrait se demander encore aujourd'hui si l'on y trouve un élargissement ou plutôt une transformation du terrain conceptuel kantien, son intention de rendre les principes de la philosophie efficaces sur le cour des hommes n'a pourtant guère changé. En effet, cette quête d'une plus grande efficacité du savoir philosophique se manifeste tout au long de son parcours dans la constante préoccupation d'en présenter les principes non seulement dans un registre scientifique, mais aussi dans un langage accessible au grand public. Cette intention se manifeste de manière paradigmatique dans la publication de la double introduction à sa Wissenschaftslehre en 1797 - la première pour les « lecteurs non-initiés » et la seconde pour ceux qui « ont déjà un système philosophique ${ }^{3} »-$ et se consolide avec la distinction élaborée

1. Lettre à Marie Johanne Rahn du 5 Septembre 1790 (Johann Gottlieb FICHTE, Gesamtausgabe der Bayerischen Akademie der Wissenschaften, hrsg. von Hans Jacob, Reinhard Lauth u. a., Stuttgart-Bad Cannstatt, Frommann-Holzboog, 1962 sq. [= GA], III, 1, p. 172).

2. GA, III, 2, p. 282.

3. GA, I, 4, p. 209. 
quelques années plus tard entre « exposé populaire », adressé au grand public, et «exposé scientifique ", adressé aux philosophes de profession ${ }^{4}$.

Le succès de cette volonté d'élargissement du rayon d'action de la philosophie dépend pourtant de la possibilité de reformuler les notions philosophiques fondamentales dans un langage accessible au plus grand nombre de lecteurs, puisqu'en effet les principes de la philosophie ne sauraient devenir efficaces sur les cœurs des hommes que si les hommes peuvent minimalement les comprendre. Il faut ainsi que les mots dont se sert la philosophie soient traduisibles dans la langue quotidienne. Les raisons profondes de cette quête de retraduction des concepts ne semblent pourtant pas se réduire à une simple prétention de divulgation ou de vulgarisation. En effet, Fichte semble parfois indiquer qu'il faut reformuler le vocabulaire de la philosophie en tant que telle. À ce propos, en janvier 1794, en pleine élaboration de son propre système qui serait annoncé quelques semaines plus tard dans l'écrit programmatique Sur le concept de la Wissenschaftslehre ou de ce que l'on appelle philosophie, il confie à Anna Henriette Schütz le besoin qu'il ressent de « donner à la philosophie un patois [Mundart] plus malléable ${ }^{5}$ ».

Cet article entend explorer l'entrecroisement de ces deux desseins : la retraduction des concepts philosophiques dans un langage populaire et la formulation d'un langage plus malléable pour la philosophie. Selon nous, une telle approche permettrait de montrer que cette quête d'un rapprochement entre langue philosophique et langue populaire s'articule à une compréhension plus générale du rapport entre philosophie et langage. Afin d'explorer cette hypothèse, notre propos suivra les étapes suivantes : (i) nous analyserons le rapport entre popularisation et flexibilisation du langage, ce qui nous permettra (ii) d'explorer les raisons systématiques du rapprochement entre langue philosophique et langue populaire et (iii) de saisir précisément la compréhension du rôle philosophique du langage qui en découle.

\section{I - Entre popularisation et provincialisation}

En avril 1806, dans la célèbre préface de L'initiation à la vie bienheureuse, Fichte annonce :

Ces leçons, jointes à celles qui viennent de paraître à la même librairie sous le titre de Caractère de l'époque actuelle [Grundzüge des gegenwärtigen Zeitalters], et à celles sur L'essence du Savant, etc. [Über das Wesen des Gelehrten und seine Erscheinungen im Gebiet der Freiheit] (Berlin, Himburg) [...] constituent l'ensemble d'un enseignement populaire dont les présentes leçons forment le sommet et le point de lumière la plus claire ${ }^{6}$.

4. GA, I, 9, p. 47.

5. Lettre du 15 janvier 1794 (GA, III, 2, p. 47-48).

6. « Diese Vorlesungen, zusammengenommen mit denen, die unter dem Titel: Grundzüge des gegenwärtigen Zeitalters, so eben in derselben Buchhandlung erschienen sind, und denen: über das 
Fichte identifie ainsi un " ensemble » d' " enseignement populaire » composé par les Grundzüge de 1804-1805, les leçons sur le savant de 1805 et L'initiation de 1806 - ce qui indique une concentration d'exposés populaires sur cette période de 1804 à $1806^{7}$. Cependant, dès lors que les leçons sur le savant de 1805 partagent la même intention que les conférences consacrées à ce même sujet en 1794 (Einige Vorlesungen über die Bestimmung des Gelehrten), elles-mêmes reprises en 1811 (Fünf Vorlesungen über die Bestimmung des Gelehrten), et dès lors que les Discours à la nation allemande (Reden an die deutsche Nation) de 1808 sont présentés comme « un prolongement des leçons » des Grundzüge ${ }^{8}$, alors on peut dire que l'enseignement populaire fichtéen se prolonge en deçà et au-delà de la période 1804-1806.

Les Discours à la nation allemande, qui s'inscrivent dans cette économie des exposés populaires, constituent un ouvrage dont la particularité ne saurait être saisie qu'en fonction du contexte de sa composition. En effet, il s'agit d'un ensemble de discours articulant un projet de résistance pour la nation allemande alors occupée depuis peu par l'armée napoléonienne ${ }^{9}$. Dans ce contexte, le but du philosophe est de persuader ses interlocuteurs de la nécessité de mettre en place le projet d' " une éducation nouvelle », dans la mesure où, selon Fichte, « le seul moyen de préserver l'existence de la nation [im Dasein zu erhalten] est la transformation complète de l'éducation ${ }^{10}$ ». Ce projet éducatif ne vise pas tant à transmettre des connaissances aux étudiants que d'inciter chez eux " l'auto-activité ${ }^{11}$ » en leur ouvrant par-là un chemin certain pour se détacher des plaisirs sensibles et pour former un ensemble éthique capable de se préserver en tant que collectivité culturelle, historique et linguistique.

L'applicabilité de ce projet éducatif dont les lignes générales sont ébauchées par Fichte dans les trois premiers des quatorze discours dépend ellemême d'un terrain fertile, c'est-à-dire de la réceptivité de la collectivité à laquelle les étudiants appartiennent par rapport à ce genre de projet. Selon le philosophe, les Allemands (les Deutsche) constitueraient une collectivité idéale pour accueillir un tel projet en vertu d'une raison fondamentale : la façon dont la langue s'est développée chez eux. On entrevoit dès lors que la langue joue un rôle essentiel dans cette nouvelle éducation et c'est précisément à ce

Wesen des Gelehrten u. s. w. (Berlin, bei Himburg) [...] machen ein Ganzes aus von populärer Lehre, dessen Gipfel, und hellsten Lichtpunkt, die gegenwärtigen bilden. » (GA, I, 9, p. 47).

7. Ce qui donne son titre au célèbre travail de Hartmut Traub : Johann Gottlieb Fichtes Populärphilosophie 1804-1806, Stuttgart, Frommann-Holzboog, 1992.

8. «Als eine Fortsetzung der Vorlesungen, die ich im Winter vor drei Jahren allhier an derselben Stätte gehalten, und welche unter dem Titel: Grundzüge des gegenwärtigen Zeitalters, gedruckt sind, habe ich die Reden, die ich hiermit beginne, angekündigt » (GA, I, 10, p. 99).

9. Dans le texte introductif rédigé par Erich Fuchs à l'édition de l'Académie, figure une reconstitution assez complète de l'atmosphère dans laquelle Fichte a prononcé ses discours : elle comprend notamment une liste des noms des auditeurs les plus importants, les dates de chaque conférence et ses répercussions politiques immédiates (GA, I, 10, p. 3-96).

10. GA, I, 10, p. 112 .

11. GA, I, 10, p. 121. 
thème que Fichte consacre une longue réflexion à la suite de la présentation générale de son projet pédagogique.

Pour Fichte, la marque distinctive d'une collectivité n'est pas tant son « lieu d'habitation [Wohnsitz] » que la langue qu'elle parle. La langue constitue un ensemble de manières d'exprimer au sein desquelles les nouvelles générations sont éduquées et à travers lesquelles elles apprennent à se rapporter à leur entourage. En se formant au sein d'une langue, l'homme accède à quelque chose «qui s'exprime par lui et se communique aux autres » et ainsi, au sens strict, il faudrait dire que « les hommes sont formés par la langue, plus que la langue ne l'est par les hommes ${ }^{12}{ }^{\prime}$. Comme nous l'avons souligné ailleurs, cette conception fichtéenne de la langue peut être associée à la notion aristotélicienne de «seconde nature » puisqu'elle est une disposition acquise, une habitude, qui devient une façon naturelle de faire, un mode naturalisé de se rapporter à autrui ${ }^{13}$.

De même que les hommes sont formés par la langue, ce n'est pas à proprement parler le peuple «qui exprime sa connaissance » à travers la langue, «mais sa connaissance qui s'exprime par lui ». C'est la langue en effet qui « donne une image sensible du suprasensible », dont la désignation se règle sur « le degré de développement qu'aura l'intelligence sensible chez ce peuple donné ». Ainsi, au moment d'exprimer des choses suprasensibles, il vaut mieux employer des mots directement issus des connaissances sensibles. Lorsqu'on le fait de cette manière, « l'image sensible [...] exprime le rapport de l'instrument spirituel à ce qui est conçu de façon entièrement compréhensible, car ce rapport s'explique à travers un autre rapport vivant à [nos] instruments sensibles ». Fichte avance ainsi que ce qui n'est pas temporel ne saurait être exprimé qu'à travers ce qui est temporel, et l'efficacité de ce mode d'expression « se règle sur l'étendue et la clarté de la connaissance sensible de celui qui le désigne ${ }^{14}$ ».

Il en découle que pour les langues ne disposant pas de rupture artificielle entre les connaissances sensibles et les modes pour se référer au suprasensible, « la clarté et la compréhensibilité immédiate des images sensibles ne sera jamais interrompue, mais elle restera un flux permanent », et le rapport au suprasensible sera d'autant plus organique et plus intuitif. Cela se manifeste, suivant l'exemple fichtéen, dans le mot ı $\delta \varepsilon ́ \alpha$ en grec ou dans le mot Gesicht en allemand : tous deux disposent d' " une signification sensible » en ce qu'ils se rapportent originellement à ce qui ne saurait être saisi que « par l'œil du

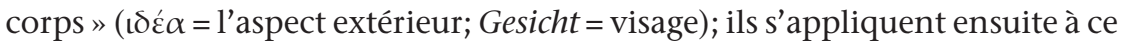

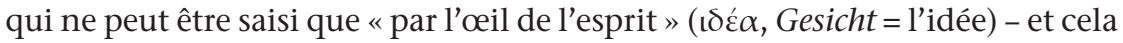
engendre une compréhensibilité plus immédiate, plus intuitive. En revanche, « lorsqu'un peuple, renonçant à sa propre langue, en adopte une autre, déjà très évoluée quant à la désignation des choses suprasensibles », ces images «au

12. GA, I, 10, p. 145-146.

13. Pour un développement plus approfondi de cette comparaison, voir Luis Fellipe GARCIA, La philosophie comme Wissenschaftslehre - le projet fichtéen d'une nouvelle pratique du savoir, Hildesheim/Zürich/ New York, Georg Olms, 2018, p. 355-359. 
lieu de s'imposer à eux avec une clarté directe et une force vitale stimulante, leur apparaîtront tout aussi arbitraires que la partie sensible de la langue ${ }^{15}$ ». Ce caractère arbitraire provoque une rupture entre « la formation spirituelle [Geistesbildung] » par laquelle on apprend à se rapporter au suprasensible (à produire des concepts) et les racines vivantes où s'inscrit le processus d'acquisition progressive de nos connaissances sensibles. En insistant sur le parallèle avec la notion aristotélicienne de seconde nature, on pourrait dire qu'en renonçant à sa propre langue au moment de se rapporter au suprasensible, un peuple, au lieu d'acquérir une disposition pour bien construire des concepts, acquiert plutôt une disposition pour mal les construire : il devient de la sorte un mauvais constructeur puisque c'est « à force de bien ou mal construire que l'on devient bon ou mauvais constructeur ${ }^{16}$ ». L'éloignement des deux registres a un effet nocif sur la construction d'images du suprasensible et donc sur le développement culturel d'un peuple.

Cet effet culturel possède encore une contrepartie sociopolitique. De fait, si les concepts sont produits dans une langue qui n'est pas la langue des connaissances sensibles quotidiennes, alors il y a un clivage entre les classes instruites et le peuple en ceci que la langue savante, celle de la formation spirituelle, devient étrangère à la langue quotidienne, celle des connaissances sensibles du peuple qui l'emploie. Comme le fait remarquer le philosophe, cette discontinuité linguistique a pour conséquence de placer une distance entre « la culture intellectuelle et la vie » de sorte que « les classes sociales qui n'ont aucun accès [à cette culture] se trouvent dans une situation d'infériorité par rapport aux classes instruites ». Cette situation est nocive non seulement pour le peuple, mais aussi pour les classes savantes puisqu'elles deviennent progressivement «comme étrangères aux autres [classes] et n'éprouvent pas le besoin de leur venir en aide » - de cela découle un « mépris pour le peuple » qui commence à " prendre des formes de plus en plus évidentes et cruelles ${ }^{17}$ ».

Cette analyse des effets culturels et sociopolitiques nocifs de l'adoption par les classes instruites d'une langue détachée de la langue quotidienne des connaissances sensibles devient d'autant plus significative qu'on la replace dans son contexte socio-culturel. En effet, une trentaine d'années avant l'invasion française et un peu plus de treize ans avant la première élaboration du projet fichtéen, le roi de Prusse, Friedrich II, déplorait publiquement - dans un essai par ailleurs paru en français sous le titre : De la littérature allemande; des défauts qu'on peut lui reprocher; quelles en sont les causes; et par quels moyens on peut les corriger - le développement limité de la langue allemande, « une langue à demi-barbare qui se divise en autant de dialectes différents que l'Allemagne contient de Provinces ${ }^{18}$ ». Le fait que le roi de Prusse exprime publiquement,

15. GA, I, 10, p. 148-150.

16. Aristote, Éthique à Nicomaque, 1103b10-11.

17. GA, I, 10, p. 163.

18. FRÉDÉRIC II, De la littérature allemande; des défauts qu'on peut lui reprocher; quelles en sont les causes; et par quels moyens on peut les corriger, Berlin, G. J. Decker, 1780, p. 6-7. 
en français, une critique de sa propre langue n'est pas un événement anodin. Bien au contraire, comme le souligne Norbert Elias, cela ne fait qu'attester sa filiation à « la société de cour », à l'aristocratie royale « dont les goûts, le style, le langage [sont] à peu de chose près les mêmes à travers toute l'Europe ${ }^{19}$ ". L'aveu du roi de Prusse ne fait ainsi que manifester un clivage très courant à l'époque, entre la langue populaire et la langue savante (le latin ou le français).

Un tel clivage a aussi des effets sur la production conceptuelle. Ainsi, Leibniz, sans doute le plus grand philosophe allemand avant la parution des Critiques kantiennes, publia ses chefs-d'œuvre (Discours de Métaphysique, Essais de Théodicée et La Monadologie) en français. De même, Christian Wolff, surnommé par Kant «le plus grand parmi les philosophes dogmatiques ${ }^{20}$ ", bien qu'il ait établi des concepts importants comme Bewusstsein et an sich dans la langue allemande, rédigea la majorité de ses travaux en latin. Cela vaut même pour Kant, dont l'ouvrage le plus important avant la parution de la Kritik der reinen Vernunft en 1781 était la dissertation De mundi sensibilis atque intelligibilis forma et principiis. On peut ainsi dire que jusqu'à la génération immédiatement antérieure à celle de Fichte, l'allemand était considéré comme une langue mineure dont les conquêtes dans le monde littéraire et philosophique étaient considérablement limitées. C'est précisément à ce moment de transition et d'articulation de nouveaux concepts dans une langue considérée comme à « demi-barbare », que Fichte annonce en janvier 1794 à Anna Schütz son intention de « donner à la philosophie un patois [Mundart] plus malléable et, en particulier, plus allemand [deutsch]».

Il est important de remarquer que le mot deutsch dérive des formes plus anciennes diutisch, diutsch, tiutsch, tiusch, utilisées à partir du $\mathrm{X}^{\mathrm{e}}$ siècle, qui sont des variantes des termes Teudo (peuple) et de l'adjectif theodisce (qui appartient au peuple, populaire) : ce mot est utilisé notamment depuis l'époque de Charlemagne pour caractériser les dialectes populaires par opposition à la langue latine officielle. D'après l'Etymologisches Wörterbuch der deutschen Sprache, ce terme était employé tantôt dans le sens de " provincial, local » tantôt dans celui d' « inculte, incapable, bête ${ }^{21}$ ». En ce sens, lorsque Fichte confie à Anna Schütz son intention de donner à la philosophie un Mundart (un patois ou une langue orale : Mundart vient de Mund - la bouche) qui soit plus deutsch justement dans un contexte sociohistorique où le latin et le français dominent encore largement le vocabulaire philosophique, il exprime sa volonté de rapprocher la langue philosophique de la langue orale des locaux (deutsche Mundart) - en d'autres termes, il veut en quelque sorte « provincialiser » la langue philosophique.

19. Norbert Elias, La civilisation des mours, trad. P. Kamnitzer, Paris, Calmann-Lévy, 1973, p. 26.

20. $\mathrm{KrV}, \mathrm{BXXXVI.}$

21. Elmar SeEbold (rév.), Kluge: Etymologisches Wörterbuch der deutschen Sprache, 24e éd., Berlin, De Gruyter, 2002, p. 371-372. Voir également l'entrée «deutsch» dans le Das Wortauskunftssystem zur deutschen Sprache in Geschichte und Gegenwart disponible en ligne : https://www.dwds.de/wb/deutsch (page consultée le 14/09/2018). 
Si l'on reprend maintenant les analyses des Discours sur la langue, on peut voir que la volonté de rendre la philosophie efficace hors de l'école passe non seulement par une traduction des concepts philosophiques dans un registre plus populaire, mais aussi par la transformation du langage philosophique en un langage capable de faire renouer la production conceptuelle (dont la visée est le suprasensible) avec le mouvement spirituel progressif de la collectivité dont il est issu (l'histoire de ses connaissances sensibles). La recherche d'une popularisation de la philosophie engendrant le besoin de traduction de son langage s'articule ici à une quête de revitalisation des outils dont se sert la philosophie : elle engendre un besoin de provincialisation de son langage afin de combattre les effets culturels et sociaux de l'éloignement entre philosophie et société.

Si cette quête de provincialisation de la langue philosophique se fonde sur le besoin d'éviter des effets pervers sur le tissu social d'un point de vue culturel et sociopolitique, il reste encore à déterminer si elle s'appuie également sur une motivation proprement philosophique. En d'autres termes : y aurait-il des raisons systématiques qui fonderaient ce besoin de provincialisation du vocabulaire conceptuel ou s'agit-il plutôt d'un besoin socio-culturel et politique lié tout simplement à l'application de la philosophie à d'autres domaines et qui ne concernerait donc pas la philosophie en tant que telle?

\section{II - La provincialisation et ses effets philosophiques}

Les Discours, tout en s'inscrivant dans l'ensemble de l'enseignement populaire en ceci qu'ils visent une reformulation des notions philosophiques dans un autre registre discursif, indiquent encore le besoin de reformuler la langue philosophique en tant que telle : une reformulation dont la nécessité se fonde sur des raisons culturelles - éviter l'éloignement de la formation spirituelle par rapport à ses racines vivantes - et sociopolitiques - combattre la fissure entre les classes instruites et le peuple. Fichte semble pourtant admettre une autre raison à ce besoin de reformulation, raison qui serait issue d'une autre sorte d'effet négatif apparaissant avec cette distance. Cet effet concerne le type de philosophie qui tend à être engendrée dans un domaine ou l'autre : d'une part, le type de philosophie tendant à se cimenter au sein des collectivités où l'on trouve un fossé linguistique entre les classes instruites et le peuple; d'autre part, le type de philosophie qui tend à se consolider au sein des collectivités où règne plutôt une continuité linguistique entre les classes.

En effet, et de façon surprenante, à la suite de l'identification des Deutsche à une collectivité idéale pour recevoir la nouvelle éducation - du fait de leur rapport plus organique et pour ainsi dire provincial à la langue (thème des quatrième et cinquième discours) - et à la suite d'une discussion concernant les raisons historiques d'une telle assertion (thème du sixième 
discours), Fichte cherche à montrer que différentes collectivités - marquées par une continuité organique ou par une discontinuité imposée entre langue savante et langage quotidien - tendent à saisir différemment l'État, la vie sociale, l'histoire, la science, la liberté et, de manière plus générale, «le principe de l'apparition » et la philosophie en tant que telle ${ }^{22}$. La différence se rapporte chaque fois au même principe général, à savoir celui suivant lequel soit les concepts sont saisis dans leur vitalité interne, soit des images rigides et fixes leur sont imposées. Dans le premier cas, il en ressort « une philosophie vraie », capable de donner libre cours au jaillissement « de la vie divine, unitaire et pure ${ }^{23}$ ». Dans le second cas apparaît une philosophie qui croit à ce qui est mort et pétrifié (todgläubige Philosophie).

Cette opposition, en ce qu'elle touche à des questions concernant la légitimité de la philosophie, semble dépasser le problème des effets - culturels et sociaux - de l'applicabilité ou de l'inapplicabilité des principes philosophiques, car il en va de la véracité même des modes distincts de philosopher, c'est-à-dire qu'il est question de la philosophie en tant que telle. Si en effet différentes collectivités, qui se distinguent précisément par leur rapport à la langue (par la continuité ou discontinuité entre les instruments sensibles de désignation et le suprasensible visé), tendent à se rapporter au savoir d'une façon telle que cela peut impacter la vérité même de la philosophie qui y est produite, alors la question centrale n'est plus simplement celle des effets socio-culturels nocifs de l'éloignement entre philosophie et société, mais plus encore, celle des effets philosophiques nocifs de celui-ci.

C'est précisément vers une évaluation de la légitimité de ces deux philosophies que le propos fichtéen semble se déployer. De fait, la todgläubige Philosophie est caractérisée comme celle dont l'assise (Grundlage) est « un être fixe [ein festes Sein] » et immuable, une philosophie pour laquelle « ce qui n'est pas une chose n'est rien du tout », de sorte que son « œil ne voit rien d'autre [que des choses] ", ne pouvant dès lors pas voir ce qui est vivant. À l'image de ces principes métaphysiques chosifiants, une telle philosophie saisit la vie sociale comme un « ordre de choses également immuable et mort », comme « une sorte de mécanisme d'horlogerie où chaque rouage, en pour-

22. La discussion de ces concepts se trouve dans le Septième Discours où Fichte soutient que la saisie $\mathrm{du}$ "principe de l'apparition [Grundlage der Erscheinung] » comme être fixe ou comme esprit (GA, I, 10, 184-186) détermine la façon dont on saisit la politique (p. 186-189), l'histoire (p. 189-190), la science (p. 191-194), soi-même (p. 194-195) et l'infini (p. 196-197). Cette thématique du rapport entre la saisie d'un principe et la vision de la multiplicité qui en est issue est récurrente chez Fichte : elle est déclinée de façon paradigmatique à la fin du deuxième exposé de la WL de 1804 ainsi que dans la sixième leçon de l'Anweisung. Une telle approche a motivé des travaux sur ce que l'on a désigné de façon perspicace comme « perspectivisme transcendantal » - voir Quentin LANDENNE, Le perspectivisme transcendantal de Fichte - les points de vue de la liberté, de la spéculation à l'application, Hildesheim/Zürich/New York, Georg Olms, 2013. Pour autant, la spécificité des Reden réside dans le fait qu'ils explorent le rôle du langage et même de la langue parlée quotidiennement, dans la saisie même de ce principe déterminant les différentes visions du monde.

23. D'après Fichte lui-même : «Die wahre in sich selbst zu Ende gekommene und über die Erscheinung hinweg wahrhaft zum Kerne derselben durchgedrungene Philosophie hingegen geht aus von dem Einen, reinen, göttlichen Leben, - als Leben schlechtweg, welches es auch in alle Ewigkeit, und darin immer Eines bleibt, nicht aber als von diesem oder jenem Leben [...] » (GA, I, 10, p. 185). 
suivant son but contribue à la marche de l'ensemble ${ }^{24}$ ». Finalement, ceux qui adoptent cette philosophie finissent par se prendre eux-mêmes pour des êtres sans vie, puisque « croire à un être fixe, immuable, inerte, c'est être mort en soi-même » et que, « dans la mesure où ils sont animés de cette croyance, [ils] deviennent semblables à ce qu'ils croient ». Une telle philosophie incapable de saisir la vie dans son mouvement est associée par Fichte à l'image d'une philosophie étrangère, une philosophie de l'être qui se trouve enfermée « dans ces ombres des ombres [...], dominée par la croyance à la mort » et qui devient par-là une philosophie qui « éprouve à l'égard de sa propre créature [ses concepts] un sentiment à la fois de crainte et d'adoration ${ }^{25}$ ».

Par opposition, la philosophie vivante est celle qui retrouve le ressort de toutes ses croyances non pas dans l'être fixe et mort, mais dans l'esprit. À l'image de cette saisie du principe comme activité vivante et vivifiante, le tissu social est lui-même compris comme ce qui est doué d'une vie interne issue de l'esprit, car c'est l'esprit même qui est pour lui « le ressort animé d'une vie propre [...] qui organise et ordonne la vie sociale ${ }^{26}$ ». La société est alors saisie comme un organisme lui-même vivant et dont la vitalité dépend de la manifestation constante de cette source vitale dans la totalité du corps social. De même, pour celui qui philosophe de cette manière, « toute existence permanente dépourvue de spiritualité n'est qu'une ombre » qu'il suffit de reconnaître comme l'expression du néant «pour s'élever à la connaissance de son propre néant et de l'Invisible comme étant la seule vérité ${ }^{27}$ ». Cette façon de philosopher est associée par Fichte à la deutsche philosophie, une philosophie qui ne reste pas enfermée dans l'être fixe, mais qui s'articule à partir de l'auto-activité originaire.

Cette opposition entre philosophie étrangère et deutsche philosophie surtout à la suite des appropriations politiques du texte fichtéen qui en ont fait un précurseur du nationalisme allemand et dont les effets dévastateurs sont bien connus ${ }^{28}$ - peut paraître douteuse, voire dangereuse. Et pourtant,

24. GA, I, 10, p. 185-186.

25. GA, I, 10, p. 197.

26. GA, I, 10, p. 188.

27. GA, I, 10, p. 198.

28. À cette appropriation idéologiquement biaisée des Discours de Fichte ont contribué, comme le fait remarquer Erich Fuchs, quelques facteurs historiques, comme le refroidissement de l'intérêt pour le système philosophique de Fichte durant les années qui ont succédé à sa mort en 1814 (découlant notamment de l'influence acquise par Schelling et Hegel sur la scène académique et du retard dans la publication des œuvres complètes de Fichte, qui paraissent seulement en 1834, soit trois ans après la mort de Hegel), puis la résurgence de l'intérêt pour ses œuvres à l'occasion des célébrations du centenaire de sa naissance en 1862, à un moment de montée du nationalisme prussien sous Bismarck, qui prend cette même année la fonction de ministre-président. La conjonction de ces deux facteurs a contribué à la réception de textes comme les Reden de façon isolée du système philosophique qui les soutient - une telle réception est ainsi passée à côté du caractère foncièrement cosmopolite traversant la philosophie fichtéenne (cf. Erich FuCHS, «Fichte - Stammvater des deutschen Nationalismus? ", Fichte-Studien, 35, Amsterdam, 2010, p. 267-284). Par ailleurs, l'essai de l'historien et futur député pro-nationaliste Heinrich von Treischke, qui associe la figure de Fichte à celle d'un «sauveur de la patrie allemande» dont le nom devrait être honoré lorsque le peuple sera prêt à «se sacrifier pour l'État », date précisément de 1862 (Heinrich von TreitsCHKE, « Fichte und die nationale Idee », in 
lorsque l'on réinscrit la démarche fichtéenne dans le contexte historique d'une occupation du territoire allemand, on peut voir que les Discours sont tout à fait étrangers à toute intention militariste d'expansion ou d'anéantissement de l'autre, puisqu'il s'agit plutôt d'un projet d'autodéfense cherchant à donner à un peuple politiquement dominé les outils pour s'approprier les façons de produire des concepts pour que les nouvelles générations puissent construire un rapport direct à l'activité savante et produire par-là une auto-conscience vivante, une philosophie vivante. Ce dont il est question dès lors, c'est d'éviter une traduction épistémique de la domination politique.

En ce sens, il serait peut-être plus éclairant de rapprocher le texte fichtéen non pas des collectivités qui, à partir d'une position de force, s'auto-affirment en écrasant leurs voisins, mais plutôt des collectivités qui, comme celle de Fichte au moment de la composition des Discours, sont en position de faiblesse, celles dont la production conceptuelle risque d'être monopolisée par une élite qui s'identifie à une façon tout à fait étrangère à sa propre collectivité d'articuler des problèmes conceptuels et qui finit " par mépriser le peuple » auquel elle appartient pourtant - en somme, des collectivités où il existe justement « un mur séparant les classes instruites du peuple ». Une telle situation se rapproche ainsi beaucoup plus de celle des pays culturellement dominés et socialement fracturés que de celle des collectivités qui se servent de leurs forces pour nourrir des projets militaristes et expansionnistes ${ }^{29}$.

En replaçant ainsi le texte dans son contexte, il en ressort une thèse originale, voire surprenante. En effet, ce projet de résistance basée sur une pédagogie qui entend rapprocher la langue savante de la langue quotidienne montre que pour combattre la fissure culturelle et sociopolitique qui sépare les classes instruites et le peuple, il est nécessaire d'instituer une éducation qui soit capable de fournir aux étudiants des instruments qu'ils puissent s'approprier pour saisir leur propre activité spirituelle. Cette saisie est précisément ce qui leur permet d'engendrer par eux-mêmes, grâce à une maîtrise progressive de leur langue - laquelle exprime une histoire de ses connaissances sensibles des images du suprasensible.

À suivre Fichte, le développement de cette capacité de production conceptuelle grâce à une sorte de provincialisation de la langue philosophique, pourrait engendrer non seulement des effets culturels (rendre le tissu social récep-

Historische und politische Aufsätze. Erster Band: Charaktere, Leipzig, 1862, p. 114-142, p. 142) - cet essai a ouvert la porte à des interprétations catastrophiques comme celles d'Ernst BERGMANN, Fichte und der Nationalsozialismus (Breslau, Hirt, 1933) ou de Carl HubER, « Fichtes nationaler Sozialismus » (Zeitschrift für Politik, 26, 1936). L'un des enjeux principaux d'une relecture des Reden comme projet pédagogique est de prendre en compte son contexte propre d'émergence, ce qui permet de montrer que les Discours, une fois replacés dans leur contexte historique, apparaissent fondamentalement comme un projet de résistance épistémique qui ne se laisse point confondre avec un quelconque expansionnisme militaire : les Discours, au contraire, prêchent la résistance. Pour une lecture nuancée des tensions internes au texte fichtéen, voir l'approche pertinente d'Étienne BALIBAR dans «Fichte et la frontière intérieure - à propos des Discours à la Nation allemande ", in Les Cahiers de Fontenay Philosophie et politique en Allemagne (XVIII - XX $X^{e}$ siècles), 58/59, Juin 1990, p. 57-82. 41/4, 2018, p. 53-74. 
tif à la formation spirituelle) et sociaux (rapprocher les classes instruites et le peuple), mais aussi des effets sur la légitimité de la façon de philosopher sur divers aspects de la vie collective (l'État, la société, l'histoire), voire sur la philosophie elle-même (la façon de saisir l'assise de l'existence). En effet, d'après Fichte, seule une philosophie qui ne travaille pas avec des principes fixes et rigides serait capable de se rapporter à ses racines vivantes, à la source originaire de l'activité. Ainsi, la rupture issue d'un problème linguistique - la distance entre langue philosophique et langue quotidienne - qui se manifeste au niveau culturel et social affecte à son tour la production épistémologique et même la philosophie en tant que telle. Une question d'abord épistémique, à savoir la langue dont on se sert pour concevoir notre entourage, se répercute au niveau social, c'est-à-dire sur le rapport entre les classes instruites et la masse, pour finalement revenir au niveau épistémique, parce qu'elle conduit à la consolidation d'une certaine façon de philosopher. En ce qui concerne la langue, il y a ainsi une sorte de détermination réciproque des niveaux épistémique et social : des transformations (bonnes ou mauvaises) sur un niveau se répercutent sur l'autre. Et c'est même en s'appuyant sur ce rapport que Fichte engendre son projet pédagogique : une éducation capable de transformer le rapport à la langue aura pour effet un réarrangement de la relation entre les classes instruites et le peuple ainsi que, sur la longue durée, un impact sur la production du savoir philosophique - ce qui aura à son tour un effet sur le rapport entre les classes instruites et le peuple, en vertu de la constitution progressive d'une collectivité plus unie, douée d'un degré plus élevé d'auto-conscience et certainement plus prête à affronter les défis de son temps.

La provincialisation de la langue philosophique peut dès lors conduire à une revitalisation sociale et épistémique, et même, sur le long terme, à l'engendrement d'une " philosophie vraie », c'est-à-dire d'une philosophie qui, parce qu'elle ne reste pas enfermée dans des principes fixes et inamovibles, s'ouvre à l'activité originaire « de la vie divine, pure et unitaire ». Mais pourquoi cette provincialisation de la langue est-elle si importante pour l'articulation d'une philosophie légitime? Quel est le rapport entre philosophie et langage qui découle de cette façon de saisir la philosophie?

\section{III - La philosophie et le langage}

Les Discours fichtéens constituent une approche du rapport entre philosophie et société qui peut être caractérisée comme une quête de provincialisation de la langue philosophique dont le but est non seulement de combattre les effets socioculturels nocifs d'un éloignement entre la langue savante et la langue quotidienne, mais aussi les effets proprement philosophiques d'une telle distance. En effet, lorsque la désignation des choses suprasensibles se fait par l'imposition arbitraire d'images issues d'autres contextes et non du déploiement interne à la langue elle-même, le libre cours de l'activité spirituelle se trouve 
paralysé par des images rigides et arbitraires. Cela veut dire que la langue est un outil et même l'outil privilégié pour parvenir à l'intuition de l'auto-activité de l'esprit - elle est un outil sensible (appartenant à l'histoire des connaissances sensibles des collectivités) pour accéder au suprasensible (l'auto-activité). Cette tension entre outil sensible et visée suprasensible indique qu'il faut, d'une part, (i) pour cerner la finalité des outils, faire la distinction entre l'intuition auto-active et les mots dont on se sert pour l'exprimer et, d'autre part, (ii) pour optimiser les outils, acquérir un vocabulaire suffisamment large pour ne pas pétrifier le dynamisme de l'auto-activité toujours dans les mêmes mots.

Le premier point indique la nécessité de ne pas surévaluer le rôle du langage, puisque « l'intuition telle que nous la concevons [justement la saisie de cette auto-activité] doit exister antérieurement à la connaissance du signe verbal $^{30}$ » : le langage doit dès lors veiller à nous reconduire, grâce à ses effets, à la saisie de ce mouvement. Fichte reste ici fidèle au principe suivant lequel il faut « laisser les mots être des mots ${ }^{31}$ ", c'est-à-dire les laisser être « des lignes auxiliaires [...] pour s'élever à l'intuition ${ }^{32}$ " qui est elle-même irréductible aux mots - c'est là précisément la raison pour laquelle la philosophie, selon la conception fichtéenne de la philosophie, «n'est pas un livre » : elle doit être constamment renouvelée "suivant chaque nouvelle condition d'époque et de communication ${ }^{33}$ ». En somme, il est important de reconnaître l'impuissance du langage face à l'intuition de l'activité.

Le second point indique qu'il est également essentiel de veiller à l'enrichissement du langage dans lequel on cherche à exprimer l'intuition. C'est pourquoi Fichte souligne l'importance primordiale au sein de l'éducation nouvelle de l'enseignement de la poésie : elle est le moyen le plus excellent (vorzüglichste) d' " introduire la pensée dans la vie commune », de nous introduire dans le domaine de ce mouvement irréductible à nos individualités de telle sorte que « le penseur est lui-même poète, dès lors qu'il désigne ses pensées dans le langage [...] en créant [des mots, des concepts] au-delà de la sphère actuelle des symboles ${ }^{34}$ ». La poésie joue ainsi le rôle fondamental d' « élar-

30. D'après Fichte, c'est la limite de la pédagogie de Pestalozzi qui, malgré sa mise en valeur de l'importance fondamentale de l'intuition pour l'apprentissage, commet l'erreur de surévaluer le pouvoir du langage (GA, I, 10, p. 217-227). Pour les notes de lectures de Fichte sur la pédagogie de Pestalozzi, voir GA, II, 10, p. 427-457.

31. Lettre à Reinhold du 2 juillet 1795 : «Wer meine Schriften studieren will, dem rate ich, Worte Worte sein zu lassen, und nur zu suchen, dass er irgendwo in die Reihe meiner Anschauungen eingreife. » (GA, III, 2, p. 344).

32. C'est la manière dont Fichte décrit le rôle des mots, du langage, dans son Rapport clair comme le jour. Il dit ainsi de celui qui se consacre à la philosophie : «Vermag er aber nicht über diese Worte, die bloße Hülfslinien sind, und über die ganze bisherige Bedeutung derselben hinweg, sich zur Sache selbst, zur Anschauung zu erheben, so mißversteht er nothwendig, auch wo er am allerbesten versteht; denn das, worauf es ankommt, ist bisher nicht gesagt, noch durch das Wort bezeichnet worden, auch läßt es sich nicht sagen, sondern nur anschauen » (GA, I, 7, p. 237).

33. «Die W. L. aber ist keinesweges ein gedruktes Buch: sondern sie ist ein lebendiger, ewig neu, $u$. frisch zu producirender Gedanke, der unter jeder andern Bedingung der Zeit, u. der Mittheilung sich anders ausspricht » (GA, II, 9, p. 181). Obigem nicht anders denn sinnbildlich geschehen kann, und zwar über den bisherigen Umkreis der 
gissement et [d']enrichissement de la sphère symbolique du langage ${ }^{35}$ ». Cet élargissement de la sphère symbolique est fondamental pour rendre le vocabulaire conceptuel plus plastique et ainsi plus prompt à ne pas endiguer l'auto-activité spirituelle dans un espace sémantique limité et rigide.

Par conséquent, une fois que la philosophie a affaire au suprasensible qu'elle ne peut exprimer qu'à travers des modes de désignations sensibles, il est d'une part nécessaire de ne pas confondre les modes de désignations sensibles, les conditions de communication de l'époque et du peuple (ou, pour utiliser une métaphore chère à Fichte : la lettre), avec ce que l'on cherche à exprimer, le suprasensible (l'esprit). D'autre part, il est également essentiel d' « élargir et compléter » le royaume des désignations sensibles, le royaume des lettres, la langue parlée par les nouvelles générations exprimant l'histoire des connaissances sensibles. En d'autres termes, il faut laisser la langue se déployer comme une langue vivante dans laquelle « les mots, ainsi que ses significations, se multiplient et changent continuellement » de façon à permettre à « celui qui l'emploie » de « la parler de façon créatrice suivant ses besoins ${ }^{36}$ ».

Ainsi, alors que Fichte cherche à rendre la philosophie efficace sur les cœurs des hommes, cette quête prend la forme non seulement d'une traduction de la langue philosophique dans la langue populaire, mais aussi celle d'une médiation du dynamisme de la vie originaire à travers les modes de désignations sensibles du langage. Pour que cette médiation puisse fonctionner, il est nécessaire d'une part de ne pas surévaluer le pouvoir du langage et, d'autre part, de rendre nos modes désignations sensibles suffisamment souples pour qu'ils puissent donner libre cours à la vie qu'ils doivent laisser passer à travers eux. Ce deuxième aspect exige justement une langue plus directement associée à l'intuition afin d'éviter la discontinuité entre les conditions de communication de son époque et les mots employés pour désigner le suprasensible. Si la langue est impuissante à exprimer l'intuition de l'auto-activité, c'est la reconnaissance de cette impuissance qui constituera alors le moteur d'une recherche de dynamisation interne à un tel instrument. La reconnaissance de l'impuissance de la langue ouvre l'horizon pour le développement de sa puissance.

On peut ainsi dire que l'efficacité de la philosophie dépend non seulement de la popularisation du langage philosophique, pour que le peuple puisse le comprendre, mais aussi d'une provincialisation de ce langage par laquelle le peuple peut se l'approprier et exprimer le dynamisme interne de l'auto-activité spirituelle, cela grâce à un outil lui-même dynamique et souple. En ce sens, le langage fonctionne comme une sorte d'outil formateur en ceci qu'il nous permet d'acquérir la flexibilité nécessaire pour laisser passer à travers nous

Sinnbildlichkeit hinaus neu erschaffend, ist Dichter » (GA, I, 10, p. 161-162).

35. GA, I, 10, p. 162.

36. Voici le passage complet: «In einer lebendigen Sprache aber, wenn nur in ihr wirklich gelebt wird, vermehren und verändern die Worte, und ihre Bedeutungen sich immerfort, und eben dadurch werden neue Zusammenstellungen möglich, und die Sprache, die niemals ist, sondern ewigfort wird, redet sich nicht selbst, sondern wer sie gebrauchen will, muss eben selber nach seiner Weise, und schöpferisch für sein Bedürfnis, sie reden » (GA, I, 10, p. 166). 
l'esprit de la vie originaire - outil qui ne peut pas nous être imposé du dehors, mais face auquel on doit pouvoir développer un rapport de continuité organique. La provincialisation de la langue philosophique entreprise par les Reden peut ainsi contribuer à l'exploration des tensions internes à la compréhension fichtéenne du langage, qui « est un outil incontournable et foncièrement inadéquat ${ }^{37}$ » du savoir philosophique, un instrument contenant simultanément une «impuissance » (Sprachohnmacht ${ }^{38}$ ) du fait de ses limites constitutives face à la vie originaire, et une " puissance » (Sprachmacht) du fait de la plasticité qu'il peut acquérir à travers le développement continu de nos langues. Si la vie unitaire que l'on cherche à exprimer ne peut pas être enfermée dans des mots rigides, on peut du moins, grâce à un enrichissement constant de notre patrimoine symbolique, ne pas l'endiguer : le monothéisme de la raison a bien besoin d'un polythéisme de l'imagination ${ }^{39}$.

l.garcia@lmu.de

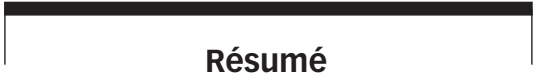

Cet article montre que Fichte développe une conception originelle du langage dans ses Discours à la Nation allemande. Il en émane non seulement le besoin de retraduire des concepts philosophiques dans une langue populaire mais aussi celui de trouver un langage plus malléable pour la philosophie en tant que telle. Afin d'explorer cette hypothèse, nous analyserons d'abord la conception fichtéenne du rapport entre popularisation et flexibilisation du langage, ce qui nous permettra d'explorer les raisons systématiques du rapprochement entre langue philosophique et langue populaire avant de resaisir avec précision la compréhension du rôle philosophique du langage qui en découle.

Mots-clés: langage, popularisation, flexibilisation, impuissance, puissance.

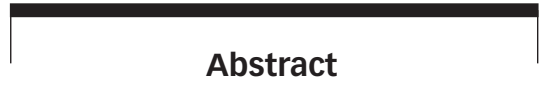

This paper demonstrates that Fichte develops an original conception of language in his Addresses to the German Nation, inasmuch as it entails the necessity of not only translating philosophical concepts into a more popular language, but also of formulating a language that is more flexible for philosophy itself. In order to explore this hypothesis, our argument will be organized in the following way: (i) an analysis of Fichte's conception of the relation between popularization and flexibilization of language, which will allow us (ii) to explore the systematic reasons for the linkage between philosophical and popular language, and (iii) to accurately understand the philosophical role of language that it entails.

Keywords: language, popularization, flexibilization, powerlessness, power.

37. Ives RADRIZZANI, «Les raisons systématiques de l'inachèvement du système fichtéen », Revista de Estud(i)os sobre Fichte, 12, 2016.

38. Christoph Asmuth, Das Begreifen des Unbegreiflichen, Stuttgart, Frommann-Holzboog, 1999, notamment «Sprachmacht und Sprachohnmacht des Philosophen », p. 153-169.

39. "Monotheismus der Vernunft und des Herzens, Polytheismus der Einbildungskraft und der Kunst, dies ist's, was wir bedürfen », voir HeGEL ou SchELling, « Das älteste Systemprogramm des deutschen Idealismus ", in Georg Wilhelm Friedrich Hegel, Werke 1 - Frühe Schriften, Suhrkamp, 1986, p. 236. 